The article appears here in its accepted form as it was provided by the submitting author. This is an Accepted Manuscript of an article published by International Journal of Sport Nutrition and Exercise Metabolism 13-04-2018 [online], available:

\title{
Development and implementation of a nutrition knowledge questionnaire for ultra-endurance athletes
}

Claire Blennerhassett

Edge Hill University, Department of Applied Health and Social Care

Lars McNaughton

Lorcan Donal Cronin

Andy Sparks

Edge Hill University, Department of Sport and Physical Activity

Correspondence concerning this article should be addressed to Claire Blennerhassett, Department Applied Health and Social Care, Edge Hill University, Ormskirk, L39 4QP, United Kingdom.

Email: claire.blennerhassett@edgehill.ac.uk 


\section{Introduction}

There has been a substantial increase in the number of recreational athletes participating in ultra-endurance races (Knetchtle et al., 2010), evidenced by an exponential increase in the number of participants in ultra-triathlons (Lenher et al., 2012) and 12-hour ultra-marathon running events (Sehovic et al., 2013). Yet, existing nutrition research has predominantly been limited to case studies (Stewart and Stewart, 2007; Bescos et al., 2012) or observational studies of the dietary practices of these athletes (Hulton et al., 2010; Black et al., 2012). This is likely due to difficulties in recruiting athletes to undertake experimental studies that meet the minimum duration for an ultra-endurance event ( $>4$ hours, Peters, 2003).

Considerable daily energy deficits have been observed for ultra-endurance runners (4732 kcals), cyclists (9915 kcals), and triathletes (5973 kcals) alike (Bescós et al., 2012; Kimber, Ross, Mason, \& Speedy, 2002; Kruseman, Bucher, Bovard, Kayser, \& Bovier, 2005). The rates of carbohydrate ingestion during ultra-endurance competitions have also been observed to be lower than 'best practice guidelines' for the discipline (90 g.hr-1 , Thomas, Erdman, \& Burke, 2016). Regarding hydration, inappropriate fluid strategies have been implicated in the development of exercise-associated hyponatraima (EAH), which is characterised by serum sodium $<135 \mathrm{mmol}^{-1}{ }^{-1}$. Recent reports have indicated that EAH affects between 4.6\% and 51.0\% (Costa et al., 2013; Hoffman et al., 2013) of ultra-endurance runners. Both inadequate nutritional intake and inappropriate fluid strategies are associated with impaired performance and negative health consequences such as nutrient deficiencies and chronic fatigue 
(Mountjoy et al., 2014). Moreover, failure to recognise the symptoms of EAH has led to a number of fatalities (Rosner \& Kirven, 2007). Therefore, assessing baseline nutrition knowledge of athletes could facilitate targeted nutrition education to optimise nutritional status during ultra-endurance training and competition.

Previous research has assessed the nutrition knowledge of athletes using sport-specific nutrition questionnaires (Jessri et al., 2010) and general nutrition questionnaires (Spendlove et al., 2012). The level of nutrition knowledge appears to vary (33.2\% to $81.8 \%$ ) within athletic populations (Trakman, 2016); however, this variability, may reflect the variety of tools used to assess nutrition knowledge and/or the athletes sources of nutrition knowledge. Jessri, et al. (2010) previously reported that those acquiring nutrition information from a

Dietitian achieved higher nutrition knowledge scores. Sport-specific questionnaires have advantages over general nutrition questionnaires when assessing the knowledge of particular athletes, as they include questions pertinent to the demands of the sport (Zinn et al., 2005). Therefore, this study aimed to develop a sport-specific nutrition questionnaire to assess the knowledge of ultra-endurance athletes.

\section{Method}

This study was conducted in two phases. Phase 1 adapted and validated a questionnaire for assessing sport and general nutrition knowledge among ultraendurance athletes. The second phase assessed the internal consistency of the questionnaire and determined the level of nutrition knowledge of a group of 
experienced ultra-endurance athletes. The study was approved by the departmental ethics committee.

\section{Phase 1 - Adaptation of a sport nutrition knowledge questionnaire Participants}

Three groups with varying levels of sports nutrition knowledge were recruited to assess the construct validity and test-retest reliability of the adapted questionnaire. These included experts from the Sports and Exercise Nutrition register (SENr; $n=10$ ) who had formal sports nutrition training, Registered Dietitians (RD; $n=10)$ with formal nutrition training for the general population, and individuals who had no nutrition education (GenP; $n=13$ ).

\section{Procedures}

Zinn and colleagues (2005) sports nutrition knowledge questionnaire was adapted for use with ultra-endurance athletes. These authors provided evidence for the content validity, construct validity and test-retest reliability of the original questionnaire with New Zealand rugby union coaches. The lead author of the current study amended the questionnaire, using literature relating to prolonged endurance research and to reflect UK nutrition products. As such, all questions and corresponding responses were scrutinised to determine their suitability for the target population. Questions relating to the nutrient composition of foods and drinks were compared to UK guidelines (e.g. $3 \mathrm{~g}$ per $100 \mathrm{~g}$ indicates low fat) and the $6^{\text {th }}$ edition of McCance and Widdoson's composition of foods integrated data set (Roe, Finglas and Church, 2007). Due to the paucity of controlled trials lasting $>4 \mathrm{hrs}$, the sport specific questions were 
considered against the demands of ultra-endurance competition and the risk associated with inappropriate nutritional intake, such as hyponatraemia. Fluid questions were amended to reflect recommendations for prolonged physical activity and questions associated with strength and power activities were removed.

The structure of the questions and the response options were also adapted to enhance the clarity and accuracy of the questionnaire. As an example, for questions relating to the level of protein contained in specific food items, the number of responses was increased from two to three, with the addition of 'medium' to the 'low' and 'high' responses available in the original questionnaire. The amended questionnaire (ULTRA-Q, see supplementary material) contained 76 questions covering the same five themes as the original questionnaire: nutrients $(n=37)$, fluid $(n=8)$, recovery $(n=11)$, body composition $(n=12)$ and supplements $(n=8)$. Examples of questions from the ULTRA-Q were as follows: "which of the following foods are high in carbohydrates" (nutrients question), "the optimum amount of fluid needed during a two-hour intense training session is:" (fluid question), "to replace energy stores, the most important nutrient to replace after a one-hour run is:" (recovery question), "if an athlete was trying to lose weight and they had the following snacks to choose from...which one should they choose?" (body composition question), and "which of the following statements are true...vitamin C should be routinely supplemented by athletes" (supplements question). Responses to questions were coded 1 (correct response) or 0 (all other responses) for statistical analysis purposes. 
Subsequently, a panel of four independent RD's with additional sport nutrition training reviewed the ULTRA-Q. They provided feedback on the clarity of the questions and the suitability of the content for ultra-endurance athletes. After amending some questions to suit the ultra-endurance domain, the panel endorsed the content validity of the questionnaire. The ULTRA-Q was then circulated electronically to the three groups for pilot testing. The purpose of the pilot testing was to assess the construct validity and test-retest reliability of the questionnaire. To facilitate this, each group completed the questionnaire on two occasions, separated by a minimum of 14 days. Electronic questionnaires were chosen for convenience, to reach a wider number of participants, and for their ability to generate comparable results to pencil-and-paper questionnaires (Lonsdale, Hodge and Rose, 2006). To improve the clarity of the questionnaire, each group was also allowed to comment on their comprehension of individual questions and provide suggestions where necessary.

\section{Data Analysis}

The data was screened for normality prior to the main statistical analysis. Skewness and kurtosis values of $<2.0$ and $<5.0$ respectively were considered to indicate reasonable normality (Tabachnick \& Fidell, 2007). Multivariate analysis of variance (MANOVA) was used to compare the total nutrition score and the five nutrition theme scores between groups. Pillai's Trace statistics were the MANOVA statistics of choice, and Scheffé's post-hoc analysis was used due to the relatively small sample size and uneven participant numbers (Tabachnick and Fidell, 2007). A statistically significant difference in the knowledge scores (total/themes) of the three groups was seen to provide 
evidence for the construct validity of the questionnaire (Litwin, 1995). An interclass correlation coefficient was computed for each of the nutrition themes to assess for test-retest reliability (Weir, 2005). A value $>0.7$ was set as the threshold for evidence of adequate test-retest reliability (Mitchell and Jolley, 2001). All data was analysed using $\mathrm{IBM}^{\odot} \mathrm{SPSS}^{\odot}$ (version 22) with a significance level of $p=0.05$ for all tests.

\section{Results}

The ULTRA-Q was completed by all participants initially and repeated by 29 $(87.9 \%)$ participants after the 14-day test-retest period. Skewness and kurtosis values ranged from -1.35 to 0.46 and -1.52 to 2.09 respectively, indicating reasonable normality (Tabachnick \& Fidell, 2007). There was a significant difference between groups for total nutrition knowledge scores, $F(10,54)=$ 9.86, $p=<0.001$, Pillai's Trace $=1.29, \eta_{p}^{2}=0.65$ (Figure 1). Post-hoc analysis revealed the SENr and RD groups scored significantly higher than GenP for nutrients, recovery, and total nutrition knowledge (Table 1). SENr scored significantly higher than RD and GenP groups for fluids and supplements knowledge. RD's were significantly higher than the GenP group for body composition knowledge. These data provided evidence for the construct validity of the questionnaire by showing that the SENr and RD groups generally scored higher than the GenP group. In terms of test-retest reliability, the intra-class correlation coefficients for the five nutrition themes (nutrients 0.95 , fluid 0.88 , recovery 0.83 , body composition 0.85 , and supplements 0.75 ) were above the threshold set $(0.70$, Weir, 2005). This provided evidence of test-retest reliability for the questionnaire. Finally, during the pilot testing, the SENr group suggested 
that contextual information could be added to the ULTRA-Q to aid in the comprehension of individual questions.

[Insert figure 1 and table 1 near here]

\section{Phase 2 - Assessing athlete's sports nutrition knowledge}

\section{Methods}

\section{Participants}

Male $(n=74)$ and female $(n=27)$ ultra-endurance athletes, aged $41.7 \pm 8.1$ and $39.0 \pm 9.6$ years respectively (Table 2 ), were recruited via a UK based research website (http://www.clultra.co.uk) to complete the finalised version of the sports nutrition knowledge questionnaire. Athletes who registered their interest were sent a link to the questionnaire (Bristol Online Survey ${ }^{\odot}$ software (Bristol, England, 2013). Two reminder emails were sent at 7 and 14 days to encourage participation.

\section{[Insert Table 2 near here]}

\section{Procedures}

After pilot testing (Phase 1), contextual information was added to the ULTRA$Q$, as recommend by the SENr group. Furthermore, eight questions were added to the questionnaire to gather demographic data and sources of nutrition knowledge, prior to completion by the ultra-endurance athletes. Like in phase 1 , responses to questions were coded $(1=$ correct response or $0=$ all other responses) for statistical analyses purposes. 
The same approach from Phase 1 was used to assess the normality of the questionnaire. The internal consistency reliability of each subscale representing the five themes was also assessed. A reliability coefficient $>0.60$ was deemed to represent adequate internal consistency reliability (Hair et al., 2006). Due to the binary nature of responses (i.e., correct or incorrect), internal consistency reliability was assessed via latent variable modelling (Raykov, Dimitrov, and Asparouhov, 2010). To compare the nutrition scores between subgroups, based on gender and ultra-endurance disciplines, a series of MANOVA's were conducted. Wilks' Lambda was the MANOVA statistic of choice due to the larger sample size (Tabachnick and Fidell, 2007) and Scheffé's post-hoc analysis was completed for its suitability when considering complicated comparisons; that is, comparing some of the disciplines and not others (Wallenstein, Zucker, \& Fleiss, 1980).

\section{Results:}

During phase 2, skewness and kurtosis values ranged from -1.42 to -0.21 and -0.91 to 3.89 respectively, indicating reasonable normality (Tabachnick \& Fidell, 2007). The reliability scores for the nutrition themes were as follows: nutrients (0.87), fluids (0.63), recovery (0.81), body composition ( 0.70$)$, and supplements (0.87). All scores were $>0.60$ (Hair et al., 2006), this provided evidence for the internal consistency reliability of each subscale of the questionnaire. The total nutrition knowledge score for all ultra-endurance athletes was $68.3 \pm 9.5 \%$, which was significantly greater than the GenP group and lower than the SENr group (Figure 1). Sub-group analysis (Table 3) based on gender revealed that the nutrition knowledge of males $(67.4 \pm 9.6 \%)$ and females $(70.7 \pm 9.3 \%)$ did 
not differ, $F(5,95)=1.73, p=0.14$, Wilks' Lambda $=0.92, \eta_{p}^{2}=0.08$. Mean nutrition scores for triathletes, cyclists, runners, and adventurers ranged between $65.1 \pm 9.4 \%$ and $72.4 \pm 8.2 \%$. There were no significant differences in nutrition knowledge between runners and triathletes, $F(5,85)=0.61, p=0.69$, Wilks' Lambda $=0.97, \eta_{p}^{2}=0.04$, but low response rates in the other disciplines prevented further comparisons. Overall, the ultra-endurance athlete's nutrition knowledge was weakest for the supplement and fluid themes. More specifically, the percentage of incorrect responses was highest $(88.1 \%)$ for the fluid question 'In an ultra-endurance race, what is the recommended amount of sodium per litre of fluid'. Similarly, only $32.7 \%$ of these athletes knew the carbohydrate concentration of sports drinks recommended for optimum hydration.

Only $5 \%$ of ultra-endurance athletes possessed a nutrition qualification (Table 2). Despite this, only $7.8 \%$ of athletes reported seeking nutrition information from a Registered Dietitian or Nutritionist (Figure 2). Other professionals involved in supporting athletes were only slightly more likely to be reported as a source of information, with $9.8 \%$ seeking nutrition advice from coaches and $11.8 \%$ from other support team members. Instead, athletes favoured nutrition information from magazines (72.5\%) and other athletes (71.6\%), with the majority of athletes (79.2\%) obtaining information from more than one source.

[Insert Table 3 near here]

[Insert Figure 2]

\section{Discussion}


Despite interest in the nutrition knowledge of athletes (Trakman et al., 2016), this is the first study to examine the knowledge of ultra-endurance athletes. Existing nutrition knowledge questionnaires lack specificity for ultra-endurance activities and were deemed unsuitable to assess the knowledge of these athletes. This study therefore employed a two-phase approach to adapt an existing questionnaire and assess the knowledge of ultra-endurance athletes. Overall, evidence was provided for the content validity, construct validity, testretest reliability, and internal consistency reliability of the ULTRA-Q. Firstly, a panel of experts provided evidence for the content validity of all items and ensured that the wording of questions was clear. Differences in nutrition knowledge scores between distinct groups (SENr, RD, and GenP) provided evidence for the construct validity of the questionnaire. Intra-class correlation coefficients between time 1 and time 2 scores provided evidence for the testretest reliability of the questionnaire. The reliability coefficients for each nutrition theme suggested that the ULTRA-Q had acceptable internal consistency reliability. Together, these findings indicate that the ULTRA-Q is an acceptable tool, which practitioners and researchers can use to assess ultra-endurance athlete's nutrition knowledge.

Overall, the total sports nutrition knowledge of the ultra-endurance athletes $(68.3 \pm 9.5 \%)$ was below the threshold $(75 \%)$ set by Torres-McGehee, et al. (2012) to demonstrate adequate knowledge. This implies that the nutrition knowledge of ultra-endurance athletes' in the present research was slightly "below par". Nevertheless, it appears that ultra-endurance athletes scored $\sim 36 \%$ higher than college athletes (Jessri et al., 2010) who completed the 
original version of the questionnaire (Zinn et al., 2005). It is possible that the superior sports nutrition knowledge of these ultra-endurance athletes may be partly attributed to their older age ( $\sim 0$ years), as acquisition of nutrition knowledge across their lifespan is likely (Wardle et al. 2000).

The importance of nutrition for ultra-endurance events is clear; therefore, perhaps these athletes are likely to make more concerted efforts to obtain nutrition information than other athletes or even coaches. Although, the majority of ultra-endurance athletes were unaware of the recommended sodium concentration of fluids for prolonged performance. This is of concern due to the potential risk of hyponatraemia during such prolonged events (Hoffman, HewButler \& Stuempfle, 2013). Furthermore, the inability to recognise the recommended carbohydrate concentration of fluids designed for optimum hydration, could have implications for gastrointestinal discomfort during competition. Hypertonic fluids have been associated with gastrointestinal symptoms that can have a detrimental impact on performance (Rehrer, Van Kemenade, Meester, Brouns, \& Saris, 1992).

Sub-group analysis in phase 2 indicated that there was no difference in nutrition knowledge between genders or between ultra-endurance disciplines. Previous studies exploring differences in nutrition knowledge between sub-groups have been equivocal (Trakman et al., 2016). This is evident, as $\sim 67 \%$ of studies exploring gender differences failed to identify any differences (Trakman et al., 2016). In contrast, Jessri et al. (2010) reported significantly higher nutrition knowledge for female athletes compared to male athletes. Whereas, Arazi and 
Hosseini (2012) reported significantly higher nutrition knowledge for male collegiate athletes compared to their non-collegiate female counterparts. Although, knowledge was comparable between male and female athletes competing at the same level (Arazi and Hosseini, 2012), suggesting that performance level may influence nutrition knowledge. The gender differences in nutrition knowledge between this study and pervious research may reflect confounding variables such as general education level, rather than gender 'per se'. The literacy level of an athlete may restrict their understanding and application of the nutrition information they obtain. Therefore, future studies using the ULTRA-Q should collect more detailed demographic information (i.e. general education level and socioeconomic status) to enable this to inform the analysis of the nutrition knowledge of these athletes.

Despite the low prevalence of nutrition qualifications amongst participants, only $8 \%$ of athletes acquired nutrition information from a Registered Dietitian/Nutritionist, compared to $74 \%$ from magazines and $73 \%$ from other athletes. Similar to the current research, Jessri et al., (2010); Shifflett et al., (2002); Sedek and Yih, (2014) report that athletes favour the media, magazines, parents, coaches, and fellow athletes as sources of nutritional knowledge, with $<1 \%$ of athletes ranking a RD in their top three sources of nutrition knowledge (Jessri, et al., 2010). This suggests that nutrition professionals need to engage in promotion activities to raise their profile amongst athletic groups. 
This study had a number of limitations, which need to be discussed. Firstly, despite rigorous promotion through social media and race websites, low responses from cyclists and adventurers prevented a comparison of these groups with runners and triathletes. Consequently, future research should look to obtain a slightly larger sample size of athletes to allow further sub-group comparisons. For a medium effect size (Cohen 1988), a priori sample size estimates, using the present test statistics, suggest that future research should recruit 128 and 135 participants for the analysis of gender and athlete group comparisons, respectively.

Secondly, the ULTRA-Q was limited to assessing the level of nutrition knowledge of ultra-endurance athletes in the UK and is not capable of determining whether a particular knowledge level translated into appropriate dietary practices. Future research assessing nutrition knowledge, alongside nutritional intake, are needed to investigate the impact of knowledge on ultraendurance athlete's dietary practices. Researchers using this questionnaire should therefore also consider the availability of the nutritional products in the athlete's country of origin, along with advances in ultra-endurance research. Additional alterations to the questionnaire may be required if new evidence alters the nutritional recommendations, or if athletes are unfamiliar with specific products.

In summary, the findings from this study provide evidence for the content validity, construct validity, test-retest reliability, and internal consistency reliability of the ULTRA-Q. As such, this questionnaire can be used to assess 
the nutrition knowledge of ultra-endurance athletes in five domains: nutrients, fluid, recovery, body composition, and supplements. In practical terms, applied practitioners could use the ULTRA-Q to assess baseline knowledge amongst ultra-endurance athletes and tailor their interventions accordingly. Future studies could also use the questionnaire alongside food/training diaries to explore the association between nutrition knowledge and energy balance within ultra-endurance athletes. 


\section{Acknowledgements}

The authors would like to thank Caryn Zinn of the Centre for Physical Activity and Nutrition, AUT University, New Zealand for kindly supplying a copy of the original sports nutrition knowledge questionnaire (Zinn et al., 2005).

\section{Authorships}

The study was designed by CB and SAS, data were collected by CB, LRM, and SAS, analyzed by CB, LRM, SAS, and LC, data interpretation and manuscript preparation were undertaken by CB, LRM, SAS and LC. All authors approved the final version of the paper.

\section{Conflicts of interest}

The authors declare they have no conflict of interest. 


\section{References}

Arazi, H., \& Hosseini, R. (2012). A comparison of nutritional knowledge and food habits of collegiate and non-collegiate athletes. SportLogia 8, 100-107.

Bescós, R., Rodríguez, F. A., Iglesias, X., Benítez, A., Marina, M., Padullés, J. M., \& Knechtle, B. (2012). High energy deficit in an ultraendurance athlete in a 24-hour ultracycling race. Proceedings (Baylor University. Medical Center), 25(2), 124-128.

Black, K. E., \& Skidmore, P. M. L. (2012). Energy intakes of ultraendurance cyclists during competition, an observational study. International Journal of Sport Nutrition and Exercise Metabolism, 22, 19-23.

Burke, L. M., Hawley, J. A., Wong, S. H. S. \& Jeukendrup, A. E. (2011). Carbohydrates for training and competition.Journal of Sports Sciences, 29, S17-S27.

Cohen J. (1988). Statistical Power Analysis for the Behavioral Sciences. New York, NY: Routledge Academic.

Costa, R. J. S., Teixeira, A., Rama, L., Swancott, A. J. M., Hardy, L. D., Lee, B., Thake, C. D. (2013). Water and sodium intake habits and status of ultraendurance runners during a multi-stage ultra-marathon conducted in a hot ambient environment: an observational field based study. Nutrition Journal, 12(1), 13. doi:10.1186/1475-2891-12-13 
Hair Jr., J., Anderson, R., Tatham, R., \& Black, W. (2006). Multivariate data analysis. USA: Pearson.

Hoffman, M. D., Hew-Butler, T., \& Stuempfle, K. J. (2013). Exercise-associated hyponatremia and hydration status in 161-km ultramarathoners. Medicine \& Science in Sports \& Exercise, 45.

Hulton, A.T., Lahart, I., Williams, K. L., Godfrey, R., Charlesworth, S., Wilson, M., Pelder, C. \& Whyte, G. (2010). Energy expenditure in the Race Across America (RAAM). International Journal of Sports Medicine 31, 463-467.

Jessri, M., Jessri, M., Rashidkhani, B. \& Zinn, C., (2010). Evaluation of Iranian College Athletes' Sport Nutrition Knowledge. International Journal of Sport Nutrition and Exercise Metabolism. 20, 257-263.

Kimbre, N. E., Ross, J. J., Mason, S. L. \& Speedy, D. B., (2002). Energy balance during an ironman triathlon in male and female triathletes. International Journal of Sports and Exercise Metabolism 12, 47-62.

Kruseman, M. Bucher, S., Bovard, M., Kayser, B. \& Bovier, P. A. (2005). Nutrient intake and performance during a mountain marathon: An observational study. European Journal of Applied Physiology 94, 151-157. 
Knechtle, B., Knechtle, P., \& Lepers, R. (2010). Participation and performance trends in ultra-triathlons from 1985 to 2009. Scandinavian Journal of Medicine and Science in Sports, 21(6), e82-90.

Litwin. M. S. (1995). How To Measure Survey Reliability and Validity. London, Sage.

Lenher, R., Knechtle, B., Rust, C. A., Rosemann, T. \& Lepers, R. (2012) From double iron to double deca iron ultra-triathlon - a retrospective data analysis from 1985 to 2011. Physical Culture and Sport. Studies and Research. 55-67.

Lonsdale, C, Hodge, K., \& Rose, E. A. (2006). Pixels vs. paper: Comparing online and traditional survey methods in sports psychology. Journal of Sporta Exercise Psychology, 28, 100-108.

Mitchell, M., \& Jolley, J. (2001). Research design explained (4th ed.). Belmont, CA:Wadsworth-Thomson Learning.

Mountjoy, M., Sundgot-Borgen, J., Burke, L., Carter, S., Constantini, N., Lebrun, C., . . L Ljungqvist, A. (2014). The IOC consensus statement: beyond the Female Athlete Triad-Relative Energy Deficiency in Sport (RED-S). British Journal of Sports Medicine, 48(7), 491-497 
Peters, E. M. (2003) Nutritional aspects in ultra-endurance exercise. Current Opinions in Clinical Nutrition and Metabolic Care. 6, 427-434

Raykov, T., Dimitrov, D. M. and Asparouhov, T. (2010). Evaluation of scale reliability with binary measures using latent variable modeling. Structural Equation Modeling, 17(2), 265-279.

Rehrer, N. J., Van Kemenade, M., Meester, W., Brouns, F., \& Saris, W. H. (1992). Gastrointestinal complaints in relation to dietary intake in triathletes. International Journal of Sport Nutrition, 2(1), 48-59.

Roe M.A., Finglas P.M, \& Church S.M. (2007) McCance and Widdowson's The Composition of Foods, 6th summary edition. The Royal Society of Chemistry, Cambridge

Rosner, M. H., \& Kirven, J. (2007). Exercise-Associated Hyponatremia. Clinical Journal of the American Society of Nephrology, 2(1), 151-161. doi:10.2215/cjn.02730806

Sedek, R. \& Yih, T.Y. (2014). Dietary habits and nutrition knowledge among athletes and non-athletes in National University Of Malaysia. Pakistan Journal of Nutrition $13,752-759$. 
Sehovic, E., Knechtle, B., Rüst, C.A. \& Rosemann, T., (2013). 12-hour ultramarathons - increasing worldwide participation and dominance of Europeans. Journal of Human Sport \& Exercise, 8(4), 932-953.

Shifflett, B., Timm, C. \& Kahanov, L. (2002). Understanding of athletes' nutritional needs among athletes, coaches and athletic trainers. Research Quarterly for Exercise and Sport. 73(3), 357-362.

Spendlove, J. L., Heaney, S. E., Gifford, J. A., Pvan, T., Denyer, G. S. \& O'Connor, H. T. (2012). Evaluation of general nutrition knowledge in elite Australian athletes British Journal of Nutrition 107, 1871-1880.

Stewart, I. B., \& Stewart, K. L. (2007). Energy balance during two days of continuous stationary cycling. Journal of the International Society of Sports Nutrition, 4, 15.

Tabachnick, B. G., \& Fidell, L. S. (2007). Using multivariate statistics (5th ed.). Boston, MA: Pearson Education Inc.

Thomas, D. T., Erdman, K. A., \& Burke, L. M. (2016). Position of the academy of nutrition and dietetics, Dietitians of Canada, and the American College of Sports Medicine: nutrition and athletic performance. Journal of the Academy of Nutrition and Dietetics, 116. doi:10.1016/j.jand.2015.12.006

Torres-Mcgehee, T. M., Pritchett, K. L., Zippel, D., Minton, D. M., Cellamare, A. 
\& Sibilia, M., (2012). Sports nutrition knowledge among collegiate athletes, coaches, athletic trainers, and strength and conditioning specialists. Journal of Athletic Training. 47(2), 205-211.

Trakman, G. L., Forsyth, A., Devlin, B. L. \& Belski, R. (2016). A systematic review of athletes' and coaches' nutrition knowledge and reflections on the quality of current nutrition knowledge measures. Nutrients 8(9) E570.

Wallenstein, S., Zucker, C. L., \& Fleiss, J. L. (1980). Some statistical methods useful in circulation research. Circulation Research, 47(1), 1-9.

Wardle, J., Parmenter, K., \& Waller, J. (2000). Nutrition knowledge and food intake. Appetite,34(3), 269-275.

Weir, J. P. (2005). Quantifying test-retest reliability using the intraclass correlation coefficient and the SEM. The Journal of Strength and Conditioning Research, 19(1), 231-240.

Zinn, C., Schofield, G. \& Wall, C., (2005). Development of a psychometrically valid and reliable sports nutrition knowledge questionnaire. Journal of Science Medicine and Sport, 8 (3) 346-351. 


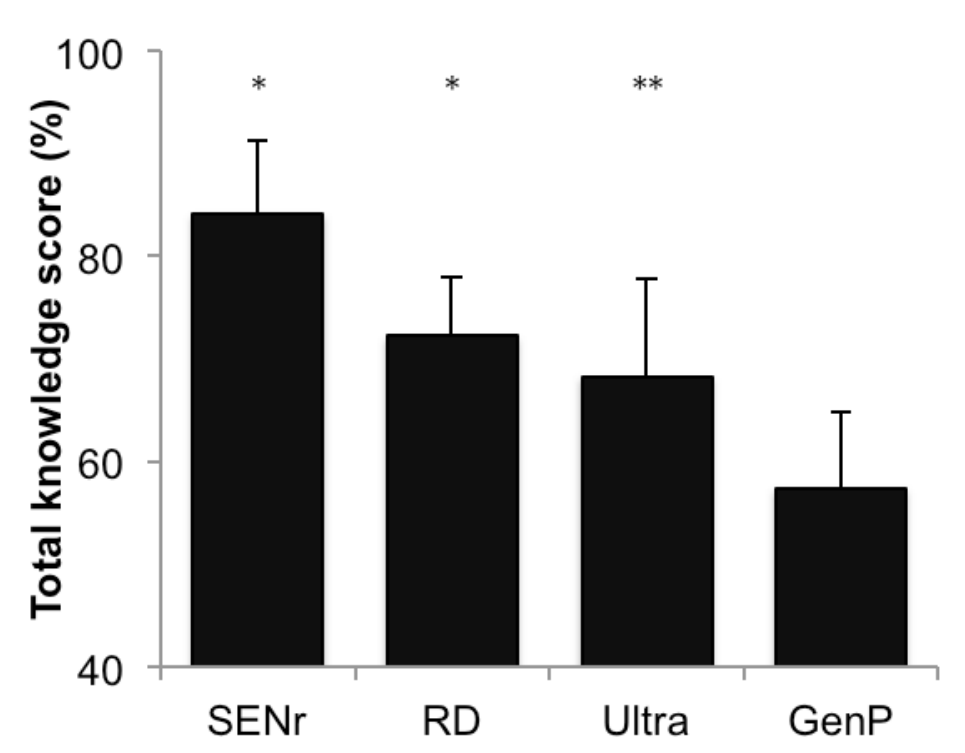

Figure 1. Nutrition knowledge of participants from phase 1 (SENr, RD and GenP) and phase 2 (ultra-endurance athletes). *SENr and RD significantly higher than GenP $(p<0.001){ }^{* *}$ Ultra-endurance athletes significantly higher than GenP and lower than SENr $(p<0.001)$. There was no difference in nutrition knowledge between RD and ultraendurance athletes 

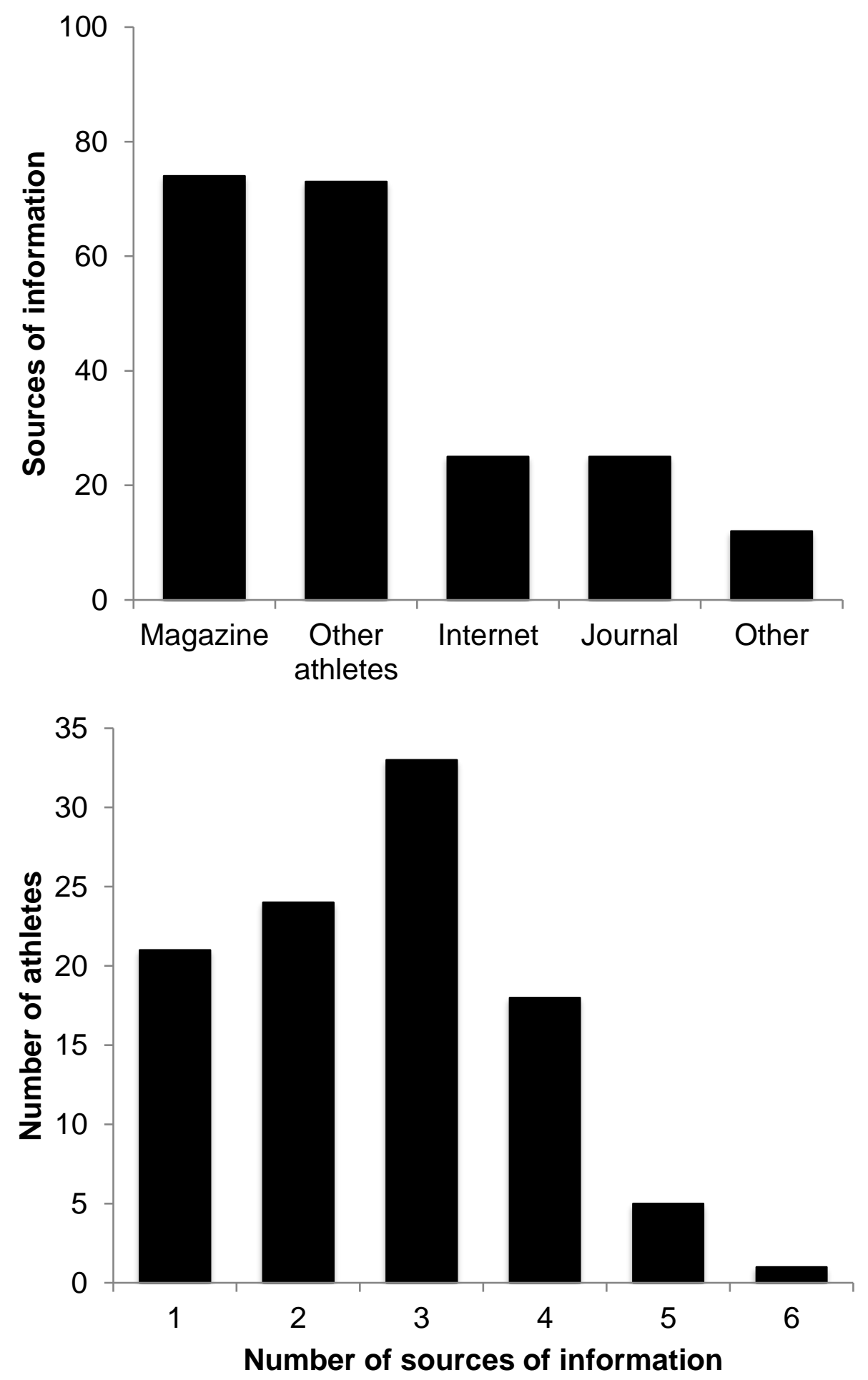

Figure 2. Key sources of nutrition information (a) and number of sources of information for ultra-endurance athletes (b). The other sources of nutrition knowledge (a) for between 2 and 12 of the ultra-endurance athletes in descending order were; support team, books, advert, coach, health professional, Registered Dietitian, trial and error, own knowledge, friend and conference. 
Table 1. Nutrition knowledge scores and sub-group analysis using Scheffé's post hoc multiple comparisons test.

\begin{tabular}{|c|c|c|c|c|c|c|}
\hline Section & SENr \% & $\mathrm{RD} \%$ & GenP \% & Comparison & Mean difference \% & Significance* \\
\hline All & $84.1 \pm 7.1$ & $76.3 \pm 5.6$ & $57.4 \pm 7.4$ & SENr - GenP & 26.7 & $<0.001$ \\
\hline Nutrients & $88.9 \pm 4.3$ & $87.8 \pm 6.9$ & $60.9 \pm 9.9$ & SENr - GenP & 28.0 & $<0.001$ \\
\hline \multirow[t]{2}{*}{ Fluid } & $76.3 \pm 1.6$ & $41.3 \pm 13.2$ & $49.0 \pm 13.2$ & SENr - RD & 35.0 & $<0.001$ \\
\hline & & & & SENr - GenP & 27.3 & 0.002 \\
\hline Recovery & $92.7 \pm 11.2$ & $89.1 \pm 8.4$ & $61.5 \pm 16.7$ & SENr - GenP & 31.2 & $<0.001$ \\
\hline Body composition & $76.7 \pm 21.1$ & $81.7 \pm 12.3$ & $62.8 \pm 13.9$ & RD - GenP & 18.9 & 0.031 \\
\hline \multirow[t]{2}{*}{ Supplements } & $68.8 \pm 19.8$ & $32.5 \pm 36.9$ & $35.6 \pm 31.4$ & $\mathrm{SENr}-\mathrm{RD}$ & 36.3 & 0.041 \\
\hline & & & & SENr - GenP & 33.2 & 0.047 \\
\hline
\end{tabular}


Table 2. Ultra-endurance athlete characteristics, and training and competition history.

\begin{tabular}{|c|c|c|}
\hline & & Athletes $n(\%)$ \\
\hline \multirow[t]{2}{*}{ Gender } & Male & 74 (73.3) \\
\hline & Female & $27(26.7)$ \\
\hline \multirow[t]{2}{*}{ Age (yrs) } & Male & $41.7 \pm 8.1^{*}$ \\
\hline & Female & $39.0 \pm 9.6^{\star}$ \\
\hline \multirow[t]{4}{*}{ Discipline } & Runner & $70(69.3)$ \\
\hline & Cyclist & $5(5.0)$ \\
\hline & Triathlete & $21(20.8)$ \\
\hline & Adventurer & $5(5.0)$ \\
\hline \multirow[t]{4}{*}{ Race locations } & UK only & $67(66.3)$ \\
\hline & Europe & $21(20.7)$ \\
\hline & USA and Canada & $3(2.9)$ \\
\hline & World & $10(9.8)$ \\
\hline \multirow[t]{4}{*}{ Total past events $(n)$} & ) $1-3$ & $34(33.7)$ \\
\hline & $4-6$ & $31(30.7)$ \\
\hline & $7-9$ & $8(7.9)$ \\
\hline & $\geq 10$ & $28(27.7)$ \\
\hline \multirow[t]{4}{*}{ Training hours } & $\leq 10$ h.week ${ }^{-1}$ & $56(55.4)$ \\
\hline & $11-20$ h.week ${ }^{-1}$ & $40(39.6)$ \\
\hline & $>20$ h.week ${ }^{-1}$ & $3(3.0)$ \\
\hline & Missing data & $2(2.0)$ \\
\hline \multirow[t]{5}{*}{ Nutrition education } & None & $96(95.0)$ \\
\hline & NVQ & $2(2.0)$ \\
\hline & Diploma & $1(1.0)$ \\
\hline & Module & $1(1.0)$ \\
\hline & Undergraduate degree & $1(1.0)$ \\
\hline
\end{tabular}

${ }^{\star}$ Mean \pm standard deviation NVQ $=$ National Vocational Qualification. 
Table 3. Nutrition knowledge score and percentage of correct responses achieved by ultra-endurance athletes.

\begin{tabular}{|c|c|c|c|c|c|c|}
\hline & Total score & Nutrients & Fluid & Recovery & Body Composition & Supplement \\
\hline & $\%$ & $\%$ & $\%$ & $\%$ & $\%$ & $\%$ \\
\hline No of questions & 76 & 37 & 8 & 11 & 12 & 8 \\
\hline All athletes & $68.3 \pm 9.5$ & $70.8 \pm 11.5$ & $58.2 \pm 18.6$ & $77.8 \pm 15.3$ & $70.1 \pm 15.4$ & $51.1 \pm 30.6$ \\
\hline Gender Males $(n=74)$ & $67.4 \pm 9.6^{*}$ & $70.2 \pm 9.3$ & $57.1 \pm 19.1$ & $76.4 \pm 16.1$ & $67.8 \pm 15.8$ & $52.4 \pm 30.6$ \\
\hline Females $(n=27)$ & $70.7 \pm 9.3^{*}$ & $72.5 \pm 12.4$ & $61.1 \pm 17.1$ & $81.5 \pm 12.7$ & $76.9 \pm 12.1$ & $47.7 \pm 30.8$ \\
\hline Significance & $p=0.14$ & & & & & \\
\hline Athlete Runner $(n=70)$ & $69.1 \pm 9.7^{\star}$ & $71.7 \pm 11.7$ & $58.6 \pm 17.4$ & $78.7 \pm 16.4$ & $71.0 \pm 14.8$ & $51.4 \pm 31.4$ \\
\hline Cyclist $(n=5)$ & $66.8 \pm 6.8$ & $62.2 \pm 9.5$ & $57.5 \pm 22.7$ & $76.4 \pm 8.1$ & $78.3 \pm 15.1$ & $67.5 \pm 16.8$ \\
\hline Triathlete $(n=21)$ & $65.1 \pm 9.4^{*}$ & $68.5 \pm 10.3$ & $53.6 \pm 21.7$ & $73.6 \pm 13.1$ & $66.3 \pm 17.7$ & $47.6 \pm 32.7$ \\
\hline Adventurer $(n=5)$ & $72.4 \pm 8.2$ & $76.8 \pm 12.5$ & $72.5 \pm 16.3$ & $83.6 \pm 13.5$ & $66.7 \pm 13.2$ & $45.0 \pm 16.8$ \\
\hline Significance & $p=0.69$ & & & & & \\
\hline
\end{tabular}

Mean \pm SD * Sub -group comparison $p>0.05$. 
Below are 20 questions assessing knowledge of a variety of sports nutrition topics, please answer them all.

\section{Nutrients}

1. Which of these foods are high in carbohydrate? (Tick one box per food).

Chicken breast

Yes No Unsure

Baked beans

$\square \quad \square$

White bread

$\square$

Butter

Cornflakes

Rice pudding

No Unsure

2. Which of these foods are low, medium and high in protein? (Tick one box per food).

Chicken breast

Low Medium High Unsure

Baked beans

$\begin{array}{ll}\square & \square \\ \square & \square \\ \square & \square \\ \square & \square \\ \square & \square \\ \square & \square\end{array}$

Apple

Margarine

Cornflakes cereal

田

$\square$

$\square$

Peanuts

3. Do you think these foods are high or low in fat? (Tick one box per food).

Avocado

High

Low

Unsure

Baked beans

च

$\square$

Pasta

Margarine

Cottage Cheese

Rice pudding

Peanuts

White bread

Honey

Hard cheese (e.g. Cheddar)

$\square \quad \square$

$\square \quad \square$

$\square$

$\square \quad \square$

$\square \quad \square$

$\square$

$\square \quad \square$

4. Which of these foods are higher or lower in saturated fat compared to unsaturated fat? (Tick one box per food). Higher in saturated fat Lower in saturated fat Unsure

Butter $\square$

Canola margarine

Whole milk

Red meat

Salmon

Chocolate

Peanuts

$\begin{array}{ll}\square & \square \\ \square & \square \\ \square & \square \\ \square & \square \\ \square & \square \\ \square & \square \\ \square & \square\end{array}$

5. Would you agree or disagree with the following statements? (Tick one box per statement). Agree Disagree Unsure

a. A high carbohydrate diet helps to reduce muscle breakdown in the body.

b. Tannins in tea decrease the amount of iron absorbed from food.

$\square$

c. Spinach is a good source of iron that is easily absorbed by the body.

d. Ascorbic acid (Vitamin C) increases the amount of iron absorbed from food. $\square$

6. Would you agree or disagree with the following statements? (Tick one box per statement). Agree Disagree Unsure

a. Whole milk contains more protein than skimmed milk.

b. Whole milk contains more calcium than skimmed milk. 
c. Green leafy vegetables contain calcium that is easily absorbed by the body.

d. Thick cut chips are a lower fat choice than thin cut chips

$\square \quad \square$

$\square$

Fluid

7.The optimum amount of fluid needed during a two-hour intense training session is: (Tick one box only.)

$1 \times 750 \mathrm{ml}$ water bottle

$3 \times 750 \mathrm{ml}$ water bottles

Athletes should create an individualised fluid plan based on sweat rate

$\square$

Unsure

8. In an ultra-endurance race, what is the recommended amount of sodium per litre of fluid? (Tick one box)

$0 \mathrm{~g}$ (it is not needed)

1-1.7 g per litre

1.7-2.9 g per litre

3-3.5 g per litre

Unsure

9. For optimum hydration, the percentage of carbohydrate in a 'sports drink' should be: (Tick one box only).

$\begin{array}{lll}5-10 \% & \square & 10-15 \% \\ 20-25 \% & \square & \text { Unsure }\end{array}$

10. Would you agree or disagree with the following statements? (Click on one box per statement).

a. Fluid loss of only $2 \%$ of an athletes body weight can reduce endurance performance especially in the heat

b. Weighing athletes before and after a training session would be a good way to determine each individual's fluid requirements.

c. Fruit juice is a good fluid to have during a training session

d. Energy drinks such as 'Red Bull' are good drinks to have 30 minutes leading up to exercise.

e. For rapid recovery between training sessions an athlete should consume 1.5 litres of fluid for every kilogram of body weight lost

Agree Disagree Unsure

Recovery

11. To replace energy stores, the most important nutrient to replace after a one-hour run is: (Tick one box only).

Carbohydrate $\quad \square \quad$ Protein

Fat $\quad \square \quad$ Unsure

12. Which one of the following set of 2 snacks would you suggest that an athlete eat after endurance training? (Tick one box for each question a-d).
a. 2 slices white bread, 2 tsp peanut butter
$\square \quad 1$ portion of chips
Unsure
b. 1 flapjack
$\square \quad 2$ sausage rolls
Unsure
c. $150 \mathrm{~g}$ pot of yoghurt
$\square \quad 2$ apples
Unsure
d. 2 low fat meat pies
$\square \quad 190 \mathrm{~g}$ pot of rice pudding $\square \quad$ Unsure

13. Which one of the following set of 2 snacks would be most effective at replacing carbohydrate stores (Tick one box for each question a-d).
a. $340 \mathrm{ml}$ can of Coke
$\square \quad 3$ cups of green salad
$\square$ Unsure
b. $1 / 2$ cup chopped dried dates
$\square \quad 1$ meat pie
Unsure
c. $180 \mathrm{~g}$ skinless chicken breast
2 slices white bread, 2 tsp marmite
$\square$ Unsure
d. $100 \mathrm{~g}$ bag of marshmallows
$\square \quad 100 \mathrm{~g}$ bag of peanut $\mathrm{M}$ and M's
$\square$ Unsure

14.When an athlete is training daily, the optimal time to eat after exercise is: (Tick one box 
only).

Between 2-3 hours

Within one hour

Within 45 minutes

Within 30 minutes

$\square$

Unsure

15. Which of these statements is the most accurate definition of the term 'Glycaemic index'. (Tick one box only.)

The amount of carbohydrate a food contains

The extent to which carbohydrate food raises blood sugar levels

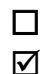

The extent to which protein food raises blood sugar levels

The extent to which carbohydrate food raises blood pressure

Unsure

Body comp

16. True or false, if exercise is unchanged, it is possible for an athlete to put on weight if they have six glasses of fruit juice in addition to their normal food intake (Tick one box only).

$\begin{array}{llll}\text { True } & \square & \text { False } & \end{array}$

17 If an athlete was trying to lose weight and they had the following snacks to choose from, which one should they choose from each line? (Tick one box for each question a-f).
a. 4 salami sticks
1 piece fruit
$\square$
Unsure $\square$
b. 2 packets of crisps
$\square 1$ cereal bar
$\square$
Unsure
c. 1 pot of rice pudding
$\square 1$ large chocolate bar
Unsure
d. $100 \mathrm{~g}$ peanuts
$\square 1$ glass of chocolate milk
$\square$
Unsure
e. 1 pot of yoghurt
$\square 1$ croissant with salad
Unsure
f. 6 crackers with cottage cheese $\nabla$
6 crackers with cheddar cheese
Unsure

18. Do you agree or disagree with the following statements? (Tick one box per statement). If an athlete wanted to lose weight, they should:

Agree Disagree Unsure

a. Exchange 1 tsp of butter on sandwiches for 1 tsp of regular margarine.

$\begin{array}{ll}\square & \square \\ \square & \square \\ \square & \square \\ \square & \square \\ \square & \square\end{array}$

b. Eat more Cheddar cheese than Edam cheese.

c. Eat less salami and more turkey breast.

d. Stop eating pasta and rice after $4 \mathrm{pm}$.

e. Exchange yoghurt, muesli bar and fruit snacks for protein shakes.

\section{Supplements}

19. Which of the following statements are true? (Tick one box per statement).

a. Vitamin $C$ should be routinely supplemented by athletes.

Agree Disagree Unsure

b. Iron tablets should be taken when an athletes feels extremely tired and is pale.

c. Multivitamin tablets should be taken by most athletes.

d. B vitamins should be taken when feeling low in energy.

e. Salt tablets should be used for athletes that get cramp during exercise.

f. Appetite suppressants are recommended for athletes with a weight loss goal.

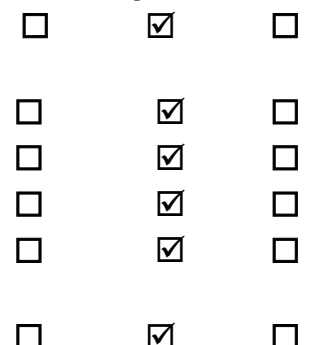

20. Do you agree or disagree with the following statements? (Tick one box per statement)

a. Sports bars can be contaminated with substances that are on the banned list from the World Anti Doping Association Agree Disagree Unsure

b. Caffeine can improve endurance performance by reducing the perception of effort.

$\nabla$

$\square$ 
Thank-you for your time, it is very much appreciated. 